\title{
Patient Characteristics and Medical Provider Factors Are Associated With Length of Stay in Total Hip Arthroplasty Patients: a Retrospective Chinese Cohort Study
}

\section{Hui Zeng ( $\square$ zenghui_36@163.com )}

Peaking University Shenzhen Hospital https://orcid.org/0000-0001-6966-0769

\section{Guoqing Li}

Peking University Shenzhen Hospital

Fei Yu

Peking University Shenzhen Hospital

Jian Weng

Peking University Shenzhen Hospital

\section{Ao Xiong}

Peking University Shenzhen Hospital

Qiang Fu

Saint Louis University

\section{Yucong Zeng}

University of lowa

\section{Fangxi Wang}

Peking University Shenzhen Hospital

\section{Chang Xu}

Peking University Shenzhen Hospital

Deli Wang

Peking University Shenzhen Hospital

\section{Research article}

Keywords: total hip arthroplasty (THA), patient characteristics, provider factors, length of stay (LOS)

Posted Date: June 23rd, 2020

DOl: https://doi.org/10.21203/rs.3.rs-35548/v1

License: (9) This work is licensed under a Creative Commons Attribution 4.0 International License. Read Full License 
Page $2 / 23$ 


\section{Abstract \\ Background}

Total hip arthroplasty (THA) is a successful treatment in the improvement of quality of life. Diagnosisrelated groups (DRGs) payment has a significant impact on the hospital market in China and length of stay (LOS) is one of its crucial manifestations. Patient characteristics and medical provider factors can affect LOS but the relationship is uncertain. We intent to explore the relationship between patient characteristics and medical provider factors and LOS of primary THA patients.

\section{Methods}

We reviewed the database containing 461 patients who underwent primary THA between January 2014 to January 2019 and regressed the LOS against a variety of perioperative factors. A multivariable linear regression model was performed to assess the difference.

\section{Results}

For parts of patient characteristics, multivariable linear regression analysis revealed that comorbidities, pre-operation albumin $<30 \mathrm{~g} / \mathrm{L}$, and pre-operation CRP $\geq 5 \mathrm{mg} / \mathrm{L}$ were all significantly associated with LOS $(p<0.05)$. For parts of medical provider factors, multivariable linear regression analysis revealed that date of surgery, urinary catheter, and incision drainage were all significantly associated with LOS ( $p<$ 0.05).

\section{Conclusions}

Patient characteristics and medical provider factors are associated with LOS of THA patients. Evaluation and identification of risk factors are beneficial in patients' education, perioperative discussion and surgery decisions in the different primary THA patient populations.

\section{Introduction}

Total hip arthroplasty (THA) is seen as a successful and effective treatment for part of improving quality of life (QOL), especially in increasing survival rates and reducing pain, for patients with hip diseases [1]. As the aged population keeps increasing, the volume of patients with hip diseases has increased substantially [2,3]. As a result, the demand for THA will also increase.

In light of the success of THA, new protocols imply that the focus of research has shifted to reduction in LOS as one of the primary outcomes of success. LOS of patients who have undergone THA can also be interpreted as an outcome measure for surgical quality. Diagnosis-related groups (DRGs) based payment 
is increasingly used in China to control hospital costs but the standards of evaluations vary. Previous review suggested that a substantial rise in the burden of osteoarthritis $(O A)$ is expected and should be addressed in health policies and DRGs payment had potential cost-saving implications by lowering LOS $[4,5]$. Extended LOS remains a paramount unsolved problem and how these different factors contribute to prolonging LOS is poorly characterized [6]. Moreover, the association between patient factors and provider factors is unclear.

Perioperative hospital adverse outcomes represent a significant origin, which were often overlooked. Even minor events such as fever or tachycardia may lead to prolonging LOS. Identifying risk factors that lengthen LOS and predicting high-risk patients before surgery is a crucial step in searching for strategies that might shorten LOS [7]. There is little literature concerning LOS after primary THA, and the specific data is insufficient.

\section{Methods}

All of the surgeries were conducted by professors who were experienced and skilled in the Department of Bone \& Joint Surgery at Peking University Shenzhen Hospital. Approaches such as direct anterior approaches, lateral, and posterior approaches were based on the general standard. The investigation of the LOS was performed carefully to ensure the validity of the study.

\section{Patients selection}

We built a dataset which contains 611 primary THA patients in our department. The mean age at surgery was 57.2 years (22 - 96 years). Data were sourced retrospectively from the discharged abstract records. Each discharged abstract involved a multitude of variables including demographic information and medical provider factors.

Patients who were lost to follow-up, refused to be discharged, underwent bilateral THA in the same period, femoral head arthroplasty, or renovation were excluded from the study. This study was a single-center, retrospective cohort study. We identified patients who met inclusion and exclusion criteria undergone primary THA by using the International Classification of Diseases 10th (ICD-10) codes. Finally, 461 patients who underwent primary THA were enrolled in this study (Fig. 1).

Study variablesWe reviewed many variables noted in the literature including a panel of perioperative variables. The variables were collected and analyzed as the follows: (1) Demographic characteristics: age, gender, body mass index (BMI); (2) Primary diagnosis: hip osteoarthritis (HOA), rheumatoid arthritis(RA), ankylosing spondylitis(AS), fracture of femoral head, femoral head necrosis, and developmental dysplaisa of the hip (DDH); (3) Comorbidities: none, hypertension, diabetes metabolic (DM), coronary heart disease (CHD), stroke, and others; (4) Preoperative hematological results: hemoglobin ( $\mathrm{Hb}$ ), albumin (Alb), C-reactive protein (CRP), and erythrocyte sedimentation rate (ESR); Change of hemoglobin $(\triangle \mathrm{Hb})$ and blood loss $(B L)$ [8], patient blood volume $(P B V)=K_{1} \times$ height $^{3}(m)+$ $\mathrm{K}_{2} \times \operatorname{mass}(\mathrm{kg})+\mathrm{K}_{3}$, (Male: $\mathrm{K}_{1}=0.3669, \mathrm{~K}_{2}=0.03219, \mathrm{~K}_{3}=0.6041 ;$ Female: $\mathrm{K}_{1}=0.3561, \mathrm{~K}_{2}=0.03308, \mathrm{~K}_{3}=$ 
0.1833), $\mathrm{BL}=\mathrm{PVB} \times($ Pre-operation hematocrit- Post-operation hematocrit). (5) Post-operative hematological results: $\mathrm{Hb}$, Alb, CRP, and ESR; (6) Provider factors: date of admission and surgery, ASA class, anesthesia method, duration of the operation, urinary catheter, and incision drainage. (7) LOS was defined as the number of days from admission to discharge.

Discharge criteria Previous studies emphasize that discharge criteria vary worldwide and suggest that patients discharged quickly tend to display a decrease in early complications following primary THA when clinically appropriate $[9,10]$. The discharge criteria in our department are as follows. (1) Patients who are willing to be discharged, (2) patients' vital signs such as appetite and sleep are normal and stable, (3) incision has healed well without any signs of infection, (4) hematological results are in normal range, (5) visual analogue scale $\leq 3$, and (6) patients who have satisfied the standard that they can walk alone with the help of aids and muscle strength grade $\geq 4$.

Statistical analysisAll statistical analyses were carried out using Stata software (version 14.0) and SPSS software (version 23.0). Continuous variables were summarized using mean \pm standard error (SE). Categorical variables were summarized using number and percent. Chi-square or Student's $t$ test were applied in univariate analysis. A linear regression model was applied to assess the difference in LOS with patient characteristics and provider variables, controlling for confounding factors in the multivariable analysis. We also reported $95 \%$ confidence interval $(C l)$ for each parameter estimate. An alpha level of 0.05 is considered statistically significant.

\section{Results}

Univariate-factor Chi-square analysis

For parts of patient demographic characteristics, the univariate-factor chi-square analysis showed no significant correlation between LOS and gender, age, BMI, or marriage $(p>0.05)$ (Table 1$)$. 
Table 1

Univariate-factor Chi-square analysis of patient demographic characteristics ( $N=461)$

\begin{tabular}{|c|c|c|c|c|c|c|c|}
\hline Factors & Classification & Number & Percentage & $\begin{array}{l}\text { Mean } \pm \\
S E\end{array}$ & $\begin{array}{l}F \\
\text { Value }\end{array}$ & $\stackrel{p}{\text { Value }}$ & $95 \% \mathrm{Cl}$ \\
\hline \multirow[t]{2}{*}{$\operatorname{Gender}\left(\mathrm{X}_{1}\right)$} & Female & 271 & $58.80 \%$ & $\begin{array}{l}17.26 \pm \\
7.09\end{array}$ & \multirow[t]{2}{*}{1.745} & \multirow[t]{2}{*}{0.08} & \multirow[t]{2}{*}{$\begin{array}{l}(-0.160- \\
2.702)\end{array}$} \\
\hline & Male & 190 & $41.20 \%$ & $\begin{array}{l}17.25 \pm \\
7.10\end{array}$ & & & \\
\hline \multirow[t]{4}{*}{ Age (year) $\left(X_{2}\right)$} & $x_{2}<60$ & 223 & $48.40 \%$ & $\begin{array}{l}17.31 \pm \\
7.15\end{array}$ & \multirow[t]{4}{*}{0.337} & \multirow[t]{4}{*}{0.74} & \multirow[t]{4}{*}{$\begin{array}{l}(-0.600- \\
0.849)\end{array}$} \\
\hline & $60 \leq X_{2}<70$ & 130 & $28.20 \%$ & $\begin{array}{l}17.27 \pm \\
7.16\end{array}$ & & & \\
\hline & $70 \leq X_{2}<80$ & 79 & $17.10 \%$ & $\begin{array}{l}17.27 \pm \\
7.11\end{array}$ & & & \\
\hline & $80 \leq x_{2}$ & 29 & $6.30 \%$ & $\begin{array}{l}17.29 \pm \\
7.14\end{array}$ & & & \\
\hline \multirow[t]{4}{*}{$\begin{array}{l}\mathrm{BMI}(\mathrm{kg} / \mathrm{m} 2) \\
\left(\mathrm{X}_{3}\right)\end{array}$} & $x_{3}<18$ & 30 & $6.50 \%$ & $\begin{array}{l}17.30 \pm \\
7.17\end{array}$ & \multirow[t]{4}{*}{-0.787} & \multirow[t]{4}{*}{0.43} & \multirow[t]{4}{*}{$\begin{array}{l}(-1.295- \\
0.554)\end{array}$} \\
\hline & $18 \leq X_{3}<24$ & 269 & $58.40 \%$ & $\begin{array}{l}17.25 \pm \\
7.10\end{array}$ & & & \\
\hline & $24 \leq X_{3}<28$ & 132 & $28.60 \%$ & $\begin{array}{l}17.25 \pm \\
7.13\end{array}$ & & & \\
\hline & $28 \leq X_{3}$ & 30 & $6.50 \%$ & $\begin{array}{l}17.34 \pm \\
7.17\end{array}$ & & & \\
\hline \multirow[t]{2}{*}{ Marriage $\left(X_{4}\right)$} & Single & 79 & $17.10 \%$ & $\begin{array}{l}17.36 \pm \\
7.20\end{array}$ & \multirow[t]{2}{*}{0.150} & \multirow[t]{2}{*}{0.88} & \multirow[t]{2}{*}{$\begin{array}{l}(-1.601- \\
1.866)\end{array}$} \\
\hline & Couple & 382 & $82.90 \%$ & $\begin{array}{l}17.25 \pm \\
7.10\end{array}$ & & & \\
\hline
\end{tabular}

For parts of patient pre-operation characteristics, the univariate-factor chi-square analysis implies that comorbidities were associated with LOS $(p<0.05)$, while primary disease, pre-operative Alb, pre-operative ESR, pre-operative CRP, or pre-operative Hb had no significant correlation with LOS $(p>0.05)$ (Table 2$)$. 
Table 2

Univariate-factor Chi-square analysis of patient pre-operation characteristics ( $\mathrm{N}=461)$

\begin{tabular}{|c|c|c|c|c|c|c|c|}
\hline Factors & Classification & Number & Percentage & $\begin{array}{l}\text { Mean } \\
\pm S E\end{array}$ & $\begin{array}{l}F \\
\text { Value }\end{array}$ & $\stackrel{p}{\text { Value }}$ & $95 \% \mathrm{Cl}$ \\
\hline \multirow[t]{5}{*}{$\begin{array}{l}\text { Primary } \\
\text { disease }\left(X_{1}\right)\end{array}$} & Degeneration & 28 & $6.10 \%$ & $\begin{array}{l}17.31 \\
\pm 7.19\end{array}$ & \multirow[t]{5}{*}{-1.656} & \multirow[t]{5}{*}{0.10} & \multirow[t]{5}{*}{$\begin{array}{l}(-1.104- \\
0.094)\end{array}$} \\
\hline & Inflammation & 56 & $12.10 \%$ & $\begin{array}{l}17.27 \\
\pm 7.11\end{array}$ & & & \\
\hline & Trauma & 152 & $33.00 \%$ & $\begin{array}{l}17.25 \\
\pm 7.11\end{array}$ & & & \\
\hline & Necrosis & 137 & $29.70 \%$ & $\begin{array}{l}17.27 \\
\pm 7.14\end{array}$ & & & \\
\hline & Deformity & 88 & $19.10 \%$ & $\begin{array}{l}17.26 \\
\pm 7.12\end{array}$ & & & \\
\hline \multirow[t]{6}{*}{$\begin{array}{l}\text { Comorbidities } \\
\left(X_{2}\right)\end{array}$} & None & 154 & $33.40 \%$ & $\begin{array}{l}15.60 \\
\pm 7.09\end{array}$ & \multirow[t]{6}{*}{3.103} & \multirow[t]{6}{*}{0.002} & \multirow[t]{6}{*}{$\begin{array}{l}(0.174- \\
0.774)\end{array}$} \\
\hline & Hypertension & 57 & $12.40 \%$ & $\begin{array}{l}16.03 \\
\pm 7.16\end{array}$ & & & \\
\hline & DM & 17 & $3.70 \%$ & $\begin{array}{l}19.29 \\
\pm 7.26\end{array}$ & & & \\
\hline & $\mathrm{CHD}$ & 46 & $10.00 \%$ & $\begin{array}{l}19.35 \\
\pm 7.00\end{array}$ & & & \\
\hline & Stroke & 36 & $7.80 \%$ & $\begin{array}{l}20.28 \\
\pm 7.17\end{array}$ & & & \\
\hline & Others & 151 & $32.80 \%$ & $\begin{array}{l}17.81 \\
\pm 7.13\end{array}$ & & & \\
\hline \multirow[t]{2}{*}{$\begin{array}{l}\text { Pre-operation Alb } \\
\left(\mathrm{X}_{3}\right)\end{array}$} & $<30 \mathrm{~g} / \mathrm{L}$ & 8 & $1.70 \%$ & $\begin{array}{l}21.38 \\
\pm 7.53\end{array}$ & \multirow[t]{2}{*}{-1.453} & \multirow[t]{2}{*}{0.10} & \multirow[t]{2}{*}{$\begin{array}{l}(-8.864- \\
1.327)\end{array}$} \\
\hline & $\geq 30 \mathrm{~g} / \mathrm{L}$ & 453 & $98.30 \%$ & $\begin{array}{l}17.18 \\
\pm 7.10\end{array}$ & & & \\
\hline \multirow[t]{2}{*}{$\begin{array}{l}\text { Pre-operation } \\
\text { ESR }\left(X_{4}\right)\end{array}$} & $<15 \mathrm{~mm} / \mathrm{h}$ & 252 & $54.70 \%$ & $\begin{array}{l}16.70 \\
\pm 7.10\end{array}$ & \multirow[t]{2}{*}{1.414} & \multirow[t]{2}{*}{0.16} & \multirow[t]{2}{*}{$\begin{array}{l}(-0.366- \\
2.245)\end{array}$} \\
\hline & $\geq 15 \mathrm{~mm} / \mathrm{h}$ & 209 & $45.30 \%$ & $\begin{array}{l}17.91 \\
\pm 7.10\end{array}$ & & & \\
\hline $\begin{array}{l}\text { Pre-operation } \\
\text { CRP }\left(X_{5}\right)\end{array}$ & $<5 \mathrm{mg} / \mathrm{L}$ & 353 & $76.60 \%$ & $\begin{array}{l}17.36 \\
\pm 7.10\end{array}$ & -1.728 & 0.09 & $\begin{array}{l}(-2.993- \\
0.192)\end{array}$ \\
\hline $\begin{array}{l}\text { For parts of provid } \\
\text { class, anesthesia, } \\
\text { admission had no }\end{array}$ & $\begin{array}{l}\text { pre-operation } \\
\text { d date of surs } \\
\text { gnificant corr }\end{array}$ & $\begin{array}{l}\text { tors, the } \\
\text { were as }\end{array}$ & $\begin{array}{l}\text { ivariate-fac } \\
\text { ciated with } L \\
S(p>0.05)\end{array}$ & $\begin{array}{l}\text { chi-squ } \\
\text { s }(p<0 .( \\
\text { able } 3) .\end{array}$ & $\begin{array}{l}\text { analys } \\
\text { while t }\end{array}$ & $\begin{array}{l}\text { implies } \\
\text { e date of }\end{array}$ & nat ASA \\
\hline
\end{tabular}




\begin{tabular}{|c|c|c|c|c|c|c|c|}
\hline Factors & Classification & Number & Percentage & $\begin{array}{l}\text { Mean } \\
\pm S E\end{array}$ & $\begin{array}{l}F \\
\text { Value }\end{array}$ & $p_{\text {Value }}$ & $95 \% \mathrm{Cl}$ \\
\hline & $\geq 5 \mathrm{mg} / \mathrm{L}$ & 108 & $23.40 \%$ & $\begin{array}{l}16.89 \\
\pm 7.12\end{array}$ & & & \\
\hline \multirow[t]{2}{*}{$\begin{array}{l}\text { Pre-operation } \mathrm{Hb} \\
\left(\mathrm{X}_{6}\right)\end{array}$} & $<110 \mathrm{~g} / \mathrm{dl}$ & 56 & $12.10 \%$ & $\begin{array}{l}19.00 \\
\pm 7.10\end{array}$ & \multirow[t]{2}{*}{-1.664} & \multirow[t]{2}{*}{0.97} & \multirow[t]{2}{*}{$\begin{array}{l}(-0.970- \\
0.081)\end{array}$} \\
\hline & $\geq 110 \mathrm{~g} / \mathrm{dl}$ & 405 & $87.90 \%$ & $\begin{array}{l}17.01 \\
\pm 7.10\end{array}$ & & & \\
\hline \multicolumn{8}{|c|}{$\begin{array}{l}S E \text { standard error; } C / \text { confidence interval; } A l b \text { albumin; } E S R \text { erythrocyte sedimentation; } C R P C \text {-reactive } \\
\text { protein; } H b \text { hemoglobin. }\end{array}$} \\
\hline \multicolumn{8}{|c|}{$\begin{array}{l}\text { For parts of provider pre-operation factors, the univariate-factor chi-square analysis implies that ASA } \\
\text { class, anesthesia, and date of surgery were associated with LOS }(p<0.05) \text {, while the date of } \\
\text { admission had no significant correlation with LOS }(p>0.05)(\text { Table } 3) \text {. }\end{array}$} \\
\hline
\end{tabular}


Table 3

Univariate-factor Chi-square analysis of provider pre-operation factors ( $\mathrm{N}=461)$

\begin{tabular}{|c|c|c|c|c|c|c|c|}
\hline Factors & Classification & Number & Percentage & $\begin{array}{l}\text { Mean } \\
\pm \text { SE }\end{array}$ & $\begin{array}{l}T / F \\
\text { Value }\end{array}$ & $p_{\text {Value }}$ & $95 \% \mathrm{Cl}$ \\
\hline \multirow[t]{4}{*}{ ASA class $\left(X_{1}\right)$} & 1 & 176 & $38.20 \%$ & $\begin{array}{l}17.27 \\
\pm 7.09\end{array}$ & \multirow[t]{4}{*}{2.195} & \multirow[t]{4}{*}{0.03} & \multirow[t]{4}{*}{$\begin{array}{l}(0.126- \\
2.280)\end{array}$} \\
\hline & 2 & 258 & $56.00 \%$ & $\begin{array}{l}17.25 \\
\pm 7.10\end{array}$ & & & \\
\hline & 3 & 24 & $5.20 \%$ & $\begin{array}{l}17.32 \\
\pm 7.20\end{array}$ & & & \\
\hline & 4 & 3 & $0.70 \%$ & $\begin{array}{l}17.26 \\
\pm 6.83\end{array}$ & & & \\
\hline \multirow[t]{2}{*}{ Anesthesia $\left(X_{2}\right)$} & General & 78 & $16.90 \%$ & $\begin{array}{l}18.74 \\
\pm 7.16\end{array}$ & \multirow[t]{2}{*}{-2.233} & \multirow[t]{2}{*}{0.03} & \multirow[t]{2}{*}{$\begin{array}{l}(-3.609- \\
-0.230)\end{array}$} \\
\hline & Local & 383 & $83.10 \%$ & $\begin{array}{l}16.95 \\
\pm 7.10\end{array}$ & & & \\
\hline \multirow[t]{6}{*}{$\begin{array}{l}\text { Date of } \\
\text { admission }\left(X_{3}\right)\end{array}$} & Weekends & 79 & $17.10 \%$ & $\begin{array}{l}17.26 \\
\pm 7.15\end{array}$ & \multirow[t]{6}{*}{-0.261} & \multirow[t]{6}{*}{0.79} & \multirow[t]{6}{*}{$\begin{array}{l}(-0.462- \\
0.354)\end{array}$} \\
\hline & Monday & 118 & $25.60 \%$ & $\begin{array}{l}17.24 \\
\pm 7.12\end{array}$ & & & \\
\hline & Tuesday & 85 & $18.40 \%$ & $\begin{array}{l}17.27 \\
\pm 7.12\end{array}$ & & & \\
\hline & Wednesday & 71 & $15.40 \%$ & $\begin{array}{l}17.29 \\
\pm 7.14\end{array}$ & & & \\
\hline & Thursday & 63 & $13.70 \%$ & $\begin{array}{l}17.26 \\
\pm 7.12\end{array}$ & & & \\
\hline & Friday & 45 & $9.80 \%$ & $\begin{array}{l}17.30 \\
\pm 7.12\end{array}$ & & & \\
\hline \multirow[t]{4}{*}{$\begin{array}{l}\text { Date of surgery } \\
\left(X_{4}\right)\end{array}$} & Weekends & 9 & $2.00 \%$ & $\begin{array}{l}17.34 \\
\pm 6.87\end{array}$ & \multirow[t]{4}{*}{-2.056} & \multirow[t]{4}{*}{0.04} & \multirow[t]{4}{*}{$\begin{array}{l}(-0.924- \\
-0.021)\end{array}$} \\
\hline & Monday & 77 & $16.70 \%$ & $\begin{array}{l}17.27 \\
\pm 7.11\end{array}$ & & & \\
\hline & Tuesday & 83 & $18.00 \%$ & $\begin{array}{l}17.26 \\
\pm 7.14\end{array}$ & & & \\
\hline & Wednesday & 88 & $19.10 \%$ & $\begin{array}{l}17.26 \\
\pm 7.12\end{array}$ & & & \\
\hline $\begin{array}{l}\text { For parts of pro } \\
\text { and transfusion } \\
\text { loss had no sigr }\end{array}$ & $\begin{array}{l}\text { surgery facto } \\
\text { associated v } \\
\text { tt correlation }\end{array}$ & $\begin{array}{l}\text { the univar } \\
\text { LOS }(p<\end{array}$ & $\begin{array}{l}\text { te-factor chi } \\
05 \text { ), while ur } \\
0.05) \text { (Table }\end{array}$ & $\begin{array}{l}\text { quare an } \\
\text { ary cath } \\
\text { ). }\end{array}$ & $\begin{array}{l}\text { sis sh } \\
\text { incis }\end{array}$ & $\begin{array}{l}\text { ed that } \\
\text { draina }\end{array}$ & $\begin{array}{l}\text { uration } \\
\text { or blood }\end{array}$ \\
\hline
\end{tabular}




\begin{tabular}{|c|c|c|c|c|c|c|c|}
\hline Factors & Classification & Number & Percentage & $\begin{array}{l}\text { Mean } \\
\pm \text { SE }\end{array}$ & $\begin{array}{l}T / F \\
\text { Value }\end{array}$ & $\stackrel{p}{\text { Value }}$ & $95 \% \mathrm{Cl}$ \\
\hline & Thursday & 100 & $21.70 \%$ & $\begin{array}{l}17.27 \\
\pm 7.16\end{array}$ & & & \\
\hline & Friday & 104 & $22.60 \%$ & $\begin{array}{l}17.26 \\
\pm 7.15\end{array}$ & & & \\
\hline \multicolumn{8}{|c|}{$S E$ standard error; $\mathrm{Cl}$ confidence interval; $A S A$ American Society of Anesthesiologists. } \\
\hline \multicolumn{8}{|c|}{$\begin{array}{l}\text { For parts of provider surgery factors, the univariate-factor chi-square analysis showed that duration } \\
\text { and transfusion were associated with LOS }(p<0.05) \text {, while urinary catheter, incision drainage, or blood } \\
\text { loss had no significant correlations with LOS }(p>0.05) \text { (Table } 4) \text {. }\end{array}$} \\
\hline
\end{tabular}


Table 4

Univariate-factor Chi-square analysis of provider surgery factors $(\mathrm{N}=461)$

\begin{tabular}{|c|c|c|c|c|c|c|c|}
\hline Factors & Classification & Number & Percentage & $\begin{array}{l}\text { Mean } \\
\pm S E\end{array}$ & $\begin{array}{l}F \\
\text { Value }\end{array}$ & $\stackrel{p}{\text { Value }}$ & $95 \% \mathrm{Cl}$ \\
\hline \multirow[t]{2}{*}{ Duration $\left(\mathrm{X}_{1}\right)$} & $<120 \min$ & 89 & $19.30 \%$ & $\begin{array}{l}16.04 \\
\pm 7.12\end{array}$ & \multirow[t]{2}{*}{2.521} & \multirow[t]{2}{*}{0.01} & \multirow[t]{2}{*}{$\begin{array}{l}(0.444- \\
3.582)\end{array}$} \\
\hline & $\geq 120 \min$ & 372 & $80.70 \%$ & $\begin{array}{l}17.54 \\
\pm 7.09\end{array}$ & & & \\
\hline \multirow[t]{2}{*}{$\begin{array}{l}\text { Urinary catheter } \\
\left(\mathrm{X}_{2}\right)\end{array}$} & No & 56 & $12.10 \%$ & $\begin{array}{l}16.25 \\
\pm 7.18\end{array}$ & \multirow[t]{2}{*}{1.644} & \multirow[t]{2}{*}{0.10} & \multirow[t]{2}{*}{$\begin{array}{l}(-0.295- \\
3.562)\end{array}$} \\
\hline & Yes & 405 & $87.90 \%$ & $\begin{array}{l}17.39 \\
\pm 7.10\end{array}$ & & & \\
\hline \multirow[t]{2}{*}{$\begin{array}{l}\text { Incision } \\
\text { drainage }\left(X_{3}\right)\end{array}$} & No & 298 & $64.60 \%$ & $\begin{array}{l}16.55 \\
\pm 7.10\end{array}$ & \multirow[t]{2}{*}{1.357} & \multirow[t]{2}{*}{0.18} & \multirow[t]{2}{*}{$\begin{array}{l}(-0.423- \\
2.311)\end{array}$} \\
\hline & Yes & 163 & $35.40 \%$ & $\begin{array}{l}18.53 \\
\pm 7.18\end{array}$ & & & \\
\hline \multirow[t]{2}{*}{ Blood loss $\left(\mathrm{X}_{4}\right)$} & No & 2 & $0.43 \%$ & $\begin{array}{l}19.67 \\
\pm 8.15\end{array}$ & \multirow[t]{2}{*}{-1.478} & \multirow[t]{2}{*}{0.14} & \multirow[t]{2}{*}{$\begin{array}{l}(-0.006- \\
0.001)\end{array}$} \\
\hline & Yes & 459 & $99.57 \%$ & $\begin{array}{l}19.67 \\
\pm 5.51\end{array}$ & & & \\
\hline \multirow[t]{2}{*}{$\begin{array}{l}\text { Transfusion } \\
\left(X_{5}\right)\end{array}$} & No & 326 & $70.70 \%$ & $\begin{array}{l}16.44 \\
\pm 7.10\end{array}$ & \multirow[t]{2}{*}{4.118} & \multirow[t]{2}{*}{$\varangle 0.001$} & \multirow[t]{2}{*}{$\begin{array}{l}(0.517- \\
1.460)\end{array}$} \\
\hline & Yes & 135 & $29.30 \%$ & $\begin{array}{l}19.20 \\
\pm 7.11\end{array}$ & & & \\
\hline \multicolumn{8}{|c|}{$S E$ standard error; $\mathrm{Cl}$ confidence interval. } \\
\hline \multicolumn{8}{|c|}{$\begin{array}{l}\text { Here are the regression curves of factors that can influence LOS in THA patients including } \\
\text { comorbidities, ASA class, anesthesia, date of surgery, duration, and transfusion (Fig. 2). }\end{array}$} \\
\hline \multicolumn{8}{|c|}{$\begin{array}{l}\text { Obviously, the results of univariate-factor analysis in this study suggest that some factors including } \\
\text { comorbidities, ASA class, anesthesia, date of surgery, duration, and transfusion are significantly } \\
\text { associated with LOS }(p<0.05) \text {, while some of the factors are not significantly statistically ( } p \otimes 0.05) \text {. } \\
\text { However, when it pertains to the literature and clinical practice, factors that are not statistically } \\
\text { significant in the univariate-factor analysis are also included in the multivariable linear regression } \\
\text { analysis. }\end{array}$} \\
\hline
\end{tabular}

\section{Multivariable linear regression analysis}

For parts of patient characteristics, multivariable linear regression analysis suggested that comorbidities (DM $(\beta=4.09), \operatorname{CHD}(\beta=2.87)$, stroke $(\beta=4.47)$, others $(\beta=1.92))$, pre-operation Alb $<30 \mathrm{~g} / \mathrm{L}(\beta=6.29)$, and pre-operation CRP $\geq 5 \mathrm{mg} / \mathrm{L}(\beta=1.94)$, are significantly associated with LOS $(p<0.05)$ (Table 5$)$. 
Table 5

Multivariable linear regression analysis of patient characteristics $(N=461)$

\begin{tabular}{|c|c|c|c|c|c|}
\hline Factors & $\beta$ & $S E$ & $T$ Value & $p$ Value & $95 \% \mathrm{Cl}$ \\
\hline \multicolumn{6}{|l|}{ Gender $\left(X_{1}\right)$} \\
\hline Female & \multicolumn{5}{|c|}{ (Reference group) } \\
\hline Male & 0.60 & 0.84 & 0.60 & 0.55 & $-1.153-2.156$ \\
\hline \multicolumn{6}{|l|}{$\operatorname{Age}\left(X_{2}\right)$} \\
\hline$x_{2}<60$ & \multicolumn{5}{|c|}{ (Reference group) } \\
\hline $60 \leq X_{2}<70$ & -1.14 & 1.01 & -1.13 & 0.26 & $-3.12-0.85$ \\
\hline $70 \leq X_{2}<80$ & -0.12 & 1.25 & -0.09 & 0.93 & $-2.57-2.33$ \\
\hline $80 \leq X_{2}$ & 1.84 & 1.78 & 1.03 & 0.30 & $-1.67-5.35$ \\
\hline \multicolumn{6}{|l|}{$\mathrm{BMI}\left(\mathrm{X}_{3}\right)$} \\
\hline$x_{3}<18$ & \multicolumn{5}{|c|}{ (Reference group) } \\
\hline $18 \leq X_{3}<24$ & 0.54 & 1.58 & 0.34 & 0.73 & $-2.56-3.63$ \\
\hline $24 \leq X_{3}<28$ & -0.06 & 1.67 & -0.04 & 0.97 & $-3.33-3.21$ \\
\hline $28 \leq X_{3}$ & -0.79 & 2.04 & -0.39 & 0.70 & $-4.81-3.23$ \\
\hline \multicolumn{6}{|c|}{ Comorbidities $\left(\mathrm{X}_{4}\right)$} \\
\hline None & \multicolumn{5}{|c|}{ (Reference group) } \\
\hline Hypertension & 0.78 & 1.25 & 0.62 & 0.53 & $-1.68-3.24$ \\
\hline DM & 4.09 & 1.99 & 2.05 & 0.04 & $0.18-8.00$ \\
\hline $\mathrm{CHD}$ & 2.87 & 1.37 & 2.09 & 0.04 & $0.17-5.57$ \\
\hline Stroke & 4.47 & 1.52 & 2.94 & 0.003 & $1.48-7.46$ \\
\hline Others & 1.92 & 0.90 & 2.13 & 0.03 & $0.15-3.70$ \\
\hline \multicolumn{6}{|c|}{ Pre-operation Alb $\left(X_{5}\right)$} \\
\hline$\geq 30 \mathrm{~g} / \mathrm{L}$ & \multicolumn{5}{|c|}{ (Reference group) } \\
\hline$<30 \mathrm{~g} / \mathrm{L}$ & 6.29 & 2.95 & 2.13 & 0.03 & $0.49-12.09$ \\
\hline \multicolumn{6}{|c|}{ Pre-operation ESR $\left(\mathrm{X}_{6}\right)$} \\
\hline$<15 \mathrm{~mm} / \mathrm{h}$ & (Refer & grou & & & \\
\hline
\end{tabular}




\begin{tabular}{|c|c|c|c|c|c|}
\hline Factors & $\boldsymbol{\beta}$ & $S E$ & $T$ Value & $p$ Value & $95 \% \mathrm{Cl}$ \\
\hline$\geq 15 \mathrm{~mm} / \mathrm{h}$ & 0.86 & 0.75 & 1.15 & 0.25 & $-0.61-2.33$ \\
\hline \multicolumn{6}{|c|}{ Pre-operation CRP $\left(X_{7}\right)$} \\
\hline$<5 \mathrm{mg} / \mathrm{L}$ & \multicolumn{5}{|c|}{ (Reference group) } \\
\hline$\geq 5 \mathrm{mg} / \mathrm{L}$ & 1.94 & 0.94 & 2.07 & 0.04 & $0.10-3.79$ \\
\hline
\end{tabular}

For parts of providers factors, multivariable linear regression analysis suggested that date of surgery (Tuesday $(\beta=-6.54)$, Wednesday $(\beta=-6.63)$, Thursday $(\beta=-5.57)$, Friday $(\beta=-6.06))$, urinary catheter $(\beta=$ $2.22)$, and incision drainage $(\beta=2.87)$ are significantly associated with LOS $(p<0.05)$ (Table 6). 
Table 6

Multivariable linear regression analysis of provider factors $(\mathrm{N}=461)$

\begin{tabular}{|c|c|c|c|c|c|}
\hline Factors & $\beta$ & $S E$ & $T$ Value & $p$ Value & $95 \% \mathrm{Cl}$ \\
\hline \multicolumn{6}{|c|}{ Anesthesia $\left(X_{1}\right)$} \\
\hline General & \multicolumn{5}{|c|}{ (Reference group) } \\
\hline Local & -1.77 & 1.01 & -1.75 & 0.08 & $-3.77-0.22$ \\
\hline \multicolumn{6}{|c|}{ Date of surgery $\left(X_{2}\right)$} \\
\hline Weekends & \multicolumn{5}{|c|}{ (Reference group) } \\
\hline Monday & -4.40 & 2.65 & -1.66 & 0.09 & $-9.62-0.82$ \\
\hline Tuesday & -6.54 & 2.67 & -2.45 & 0.01 & $-11.78--1.29$ \\
\hline Wednesday & -6.63 & 2.65 & -2.50 & 0.01 & $-11.83--1.42$ \\
\hline Thursday & -5.57 & 2.66 & -2.10 & 0.04 & $-10.80--0.36$ \\
\hline Friday & -6.06 & 2.64 & -2.29 & 0.02 & $-11.26--0.86$ \\
\hline \multicolumn{6}{|c|}{ Urinary catheter $\left(X_{3}\right)$} \\
\hline No & \multicolumn{5}{|c|}{ (Reference group) } \\
\hline Yes & 2.22 & 1.13 & 1.96 & 0.04 & $0.001-4.43$ \\
\hline \multicolumn{6}{|c|}{ Incision drainage $\left(X_{4}\right)$} \\
\hline No & \multicolumn{5}{|c|}{ (Reference group) } \\
\hline Yes & 2.87 & 0.79 & 3.65 & $<0.001$ & $1.32-4.41$ \\
\hline
\end{tabular}

\section{Discussion}

Lots of relevant research has consistently identified THA as a successful treatment for patients' endstage hip diseases [11]. Over time, the implementation process of DRGs-based payment has developed rapidly and LOS has become one of the most critical manifestations of health policies [12, 13]. Even though there has been rapid progress in THA surgery, there is little literature concerning factors that may affect LOS of THA patients. Peters et al believed that factors could be classified into two categories: patient characteristics and medical provider factors [14]. The concept of LOS was investigated quite intensively in recent years, but the reasons for extending LOS are currently unknown.

Although former researchers have spent much effort on shortening LOS and improving outcomes of THA. Unfortunately, most of the findings are varied and uncertain and they have not developed an efficient and 
effective method yet. Accurate data, regarding risk factors for prolonging LOS after THA, are critical for improving health outcomes. Efforts to reduce LOS and minimize adverse outcomes are necessary. Therefore, it is significant to explore the influence of the factors.

Reports from international registries revealed that extended LOS was independently associated with a history of comorbidities such as diabetes, heart disease, and hypertension, which were correlated with improved selected outcomes $[15,16]$. Our findings are in consistent with earlier reports that comorbidity is one of the risk factors. For future patient care, multiple disciplinary discussions and more specifically perioperative evaluation are necessary for patients who were caught comorbidity if possible.

Previous papers demonstrated that several pre-operative variables were independently associated with extended LOS. Lower pre-operative Alb is one of the independent risk factors for predicting adverse outcomes following primary THA [17-19]. Our findings are in line with earlier reports and confirm the strong association between LOS and hypoalbuminemia (Alb $<30 \mathrm{~g} / \mathrm{L}$ ). Pre-operative Alb could promote incision healing and reflect the nutrition status, while hypoalbuminemia would lead to a variety of complications or even death. Malnutrition is a modifiable risk factor for poor outcomes and surgeons should pay close attention to patients' nutrition status. Accurate identification of patients' malnutrition will allow pre-operative nutrition interventions, which can help to improved post-operative outcomes.

Besides hypoalbuminemia, researchers demonstrated that abnormal pre-operative hematological tests are associated with prolonged LOS [20]. Lower pre-operative health status was associated with less satisfaction [21]. Our findings are in consistent with earlier reports and confirm the strong association between pre-operation CRP and LOS. CRP is a sensitive indicator predicting the probability of infection and inflammation. Laboratory evaluation could help surgeons identify and take appropriate preventive measures for high-risk patients. They can also help guide preparation pre-operatively and determine the intervention of routine follow-up post-operatively.

For patient characteristics, comorbidity is a non-modifiable risk factor but the status of nutrition and hematological results are modifiable. Devoting more attention to selected patients emphasizes the importance of considering comorbidities and preparing positive interventions in the context of the specific procedure.

Previous studies reported that surgical techniques could reduce adverse outcomes [22]. Acute nerve injury is rare but potentially devastating following THA due to the schedule of surgery[23]. Our results are consistent with earlier reports and confirm the strong associations linking LOS to date of surgery. These associations imply that schedule of operation is significant and crucial variables in improving outcomes. Surgical techniques and proper schedule are needed to be improved in order to perform surgery accurately.

Routine use of urinary catheterization in fast-track THA may increase the risk of postoperative urinary retention and infection, which are potential sources of infection [24]. These findings conflict against the routine use of perioperative indwelling catheterization $[25,26]$. However, our results imply that 
catheterization is a risk factor and confirm the strong association between catheterization and LOS. Former research findings are debatable and further study may be needed. Patients treated with indwelling catheterization, history of urinary retention, and high volumes of fluid volume were significantly more likely to experience urinary retention and infection postoperatively. Intermittent catheterization, or removing it in the early stage, would be better choices for selected patients to prevent adverse outcomes if necessary.

Several papers have used administrative databases to evaluate drainage, which was associated with a prolonged LOS $[27,28]$. Drainage could result into heterotopic ossification, which may result in more complications due to restriction of early postoperative mobilization and exercise [29, 30]. All of these findings are in line with our results and we indicate that drainage is a risk factor of prolonged LOS. Furthermore, no-drainage for easy THA may be a better choice for surgeons but complicated THA should be evaluated in details.

Based on the current finding, we can draw the conclusion that schedule of surgery, urinary catheter, and drainage can extend LOS for THA patients for parts of medical care providers. This knowledge not only can help physicians guide patients' expectations before THA but also direct surgeons devoting more attention to these factors in case of bringing negative effect.

Additionally, previous analysis demonstrated that extended LOS was independently associated with gender and age, and obesity [31,32]. Some research believes that understanding current practice patterns in anesthesia may serve as a platform for future work aimed at maximizing effective postoperative pain control and minimizing the risks $[33,34]$. The former research findings are not in line with ours but our data revealed the tendency about this, which may be due to the limitation of our dataset and the patient characteristics of our registration. We will consider increasing our sample size and performing a followup investigation in a future study.

Previous studies have emphasized potential advantages in reducing LOS. Evaluation of risk factors is beneficial in discussion of the perioperative risks of THA and patients' education in the different patient populations, and future studies should try to identify more precise factors. In order to shorten the LOS and improve THA outcomes, surgeons should pay more attention to selected patients during the perioperative management. Individualized treatment schedules and proper methods are needed to strengthen the intervention, which would be highly beneficial in shortening the LOS.

Furthermore, there are several limitations in our research study. First, we conducted this retrospective cohort study by using a database in our department several years ago, particularly with new generation constructs. Prospective studies are needed to validate these calculators and to refine them over time. Moreover, the standard of discharge may not be consistent according to the surgeon. Last but not least, the database did not include information on long-term follow-up outcomes. We believe that future studies are necessary to define the risk factors clearly and use more robust experimental designs to confirm the results. 


\section{Conclusions}

The study explores risk factors and illustrates the role of predicting the occurrence of prolonging and shortening the LOS in primary THA patients. For patient characteristics, comorbidities, pre-operation Alb < $30 \mathrm{~g} / \mathrm{L}$, and pre-operation CRP $\geq 5 \mathrm{mg} / \mathrm{L}$ may prolong LOS. For provider variables, duration and schedule of surgery, urinary catheter, and incision drainage may prolong LOS. Our study is useful for identifying risk factors of both patients and providers, which can help shorten the LOS, make better perioperative plans and improve the quality of medical care.

\section{Abbreviations}

THA

Total hip arthroplasty; LOS:Length of stay; DRGs:Diagnosis-related groups; OA:Osteoarthritis; QOL:quality of life; ASA:American Society of Anesthesiologists; BMI:Body mass index; SE:standard error; Cl:Confidence intervals; CRP:C-reactive protein; Hb:Hemoglobin; Alb:albumin; ESR:Erythrocyte sedimentation rate; RA:Rheumatoid arthritis; AS:Ankylosing spondylitis; DDH:Developmental dysplaisa of the hip; DM:Diabetes metabolic; CHD:coronary heart disease; ICD-10:International Classification of Diseases 10th. BL:blood loss; PBV:patient blood volume.

\section{Declarations}

\section{Availability of data and materials}

Please contact the author for data requests.

\section{Ethics approval and consent to participate}

Ethical review approval was obtained from the Human Subject Committee at Ethics Committee of Peking University Shenzhen Hospital (Ethics Committee of Peking University Shenzhen Hospital (research) [2020] 013th).

\section{Conflict of interest statement:}

All of the authors of this paper have disclosed potential or pertinent conflicts of interest, which may include receipt of payment, either direct or indirect, institutional support, or association with an entity in the biomedical field which may be perceived to have potential conflict of interest with the submitted article. Each author certifies that his or her institution approved the human protocol for this investigation and that all investigations were conducted in conformity with ethical principles of research.

\section{Funding:}


This study was supported by grants from "San-Ming" Project of Medicine in Shenzhen (No.

SZSM201612092), Natural Science Foundation of Guangdong Province (No. 2019A1515011290) and Research and Development Projects of Shenzhen (No. JCYJ20170307111755218).

\section{Authors' contributions:}

Guoqing Li, Fei Yu, and Jian Weng reviewed and collected the database, conceived the design of the study, and prepared the manuscript. Guoqing Li and Fangxi Wang collected and inputted the data. Chang Xu and Qiang Fu carried out the statistical analysis. Qiang Fu, Deli Wang, Ao Xiong, and Yucong Zeng assisted with the revision of the manuscript. Chang Xu, Deli Wang, Hui Zeng initiated and designed the study. All authors read and approved the final manuscript.

\section{Acknowledgements}

We thank the entire staff of the Department of Bone \& Joint Surgery in Peking University Shenzhen Hospital, who give their assistance throughout the course of this study.

\section{References}

1. Bovonratwet, P., M.L. Webb, N.T. Ondeck, A.M. Lukasiewicz, J.J. Cui, R.P. McLynn, and J.N. Grauer, Definitional Differences of 'Outpatient' Versus 'Inpatient' THA and TKA Can Affect Study Outcomes. Clin Orthop Relat Res, 2017. 475(12): p. 2917-2925.

2. Streit, M.R., B. Lehner, D.S. Peitgen, M.M. Innmann, G.W. Omlor, T. Walker, C. Merle, and B. Moradi, What Is the Long-term (27-to 32-year) Survivorship of an Uncemented Tapered Titanium Femoral Component and Survival in Patients Younger Than 50 Years? Clin Orthop Relat Res, 2020.

3. Lovald, S.T., K.L. Ong, A.L. Malkani, E.C. Lau, J.K. Schmier, S.M. Kurtz, and M.T. Manley, Complications, mortality, and costs for outpatient and short-stay total knee arthroplasty patients in comparison to standard-stay patients. J Arthroplasty, 2014. 29(3): p. 510-5.

4. Meng, Z., W. Hui, Y. Cai, J. Liu, and H. Wu, The effects of DRGs-based payment compared with costbased payment on inpatient healthcare utilization: A systematic review and meta-analysis. Health Policy, 2020.

5. Kiadaliri, A.A., L.S. Lohmander, M. Moradi-Lakeh, I.F. Petersson, and M. Englund, High and rising burden of hip and knee osteoarthritis in the Nordic region, 1990-2015. Acta Orthop, 2018. 89(2): p. 177-183.

6. Hsiue, P.P., C.J. Chen, C. Villalpando, D. Ponzio, A. Khoshbin, and A.I. Stavrakis, Trends and patient factors associated with technology-assisted total hip arthroplasty in the United States from 2005 to 2014. Arthroplast Today, 2020. 6(1): p. 112-117.e1.

7. Kawai, T., M. Kataoka, K. Goto, Y. Kuroda, K. So, and S. Matsuda, Patient- and Surgery-Related Factors that Affect Patient-Reported Outcomes after Total Hip Arthroplasty. J Clin Med, 2018. 7(10). 
8. Gross, J.B., Estimating allowable blood loss: corrected for dilution. Anesthesiology, 1983. 58(3): p. 277-80.

9. Mayer, M.A., K. Pirruccio, M. Sloan, and N.P. Sheth, Discharge Home is Associated With Decreased Early Complications Following Primary Total Joint Arthroplasty. J Arthroplasty, 2019.

10. Malahias, M.A., A. Gu, S.S. Richardson, I. De Martino, M.P. Ast, and P.K. Sculco, Hospital Discharge Within a Day After Total Hip Arthroplasty Does Not Compromise 1-Year Outcomes Compared With Rapid Discharge: An Analysis of an Insurance Claims Database. J Arthroplasty, 2019.

11. Sikora-Klak, J., A. Gupta, C. Bergum, B. Zarling, and D.C. Markel, The Evaluation of Comorbidities Relative to Length of Stay for Total Joint Arthroplasty Patients. J Arthroplasty, 2017. 32(4): p. 10851088.

12. Siam, K., N. Roeder, U.R. Folsch, and H.F. Spies, [Representation of Internal Medicine in G-DRG System - Analysis of Reasons for Prolonged Length of Stay]. Dtsch Med Wochenschr, 2017. 142(15): p. 11441152.

13. Almaguer, A.M., A.R. Hsu, J.M. Pearson, H.M. McKissack, J.R. Jones, S.M. Naranje, and A.B. Shah, Do Geographic Region, Medicaid Status, and Academic Affiliation Affect Access to Care Among Medicaid and Privately Insured Total Hip Arthroplasty Patients? J Arthroplasty, 2019.

14. Peters, R.M., L.N. van Steenbergen, R.E. Stewart, M. Stevens, P.C. Rijk, S.K. Bulstra, and W.P. Zijlstra, Patient Characteristics Influence Revision Rate of Total Hip Arthroplasty: American Society of Anesthesiologists Score and Body Mass Index Were the Strongest Predictors for Short-Term Revision After Primary Total Hip Arthroplasty. J Arthroplasty, 2019.

15. Malabu, U.H., O. Adegboye, O.G. Hayes, A. Ryan, V.N. Vangaveti, S. Jhamb, K. Robertson, and K.S. Sangla, Influence of Ethnicity on Outcomes of Diabetes Inpatient Hypoglycemia: an Australian Perspective. J Endocr Soc, 2020. 4(2): p. bvaa009.

16. Lan, R.H. and A.F. Kamath, Does the Timing of Pre-Operative Medical Evaluation Influence Perioperative Total Hip Arthroplasty Outcomes? Open Orthop J, 2017. 11: p. 195-202.

17. Li, G., J. Weng, C. Xu, D. Wang, A. Xiong, and H. Zeng, Factors associated with the length of stay in total knee arthroplasty patients with the enhanced recovery after surgery model. J Orthop Surg Res, 2019. 14(1): p. 343.

18. Rudasill, S., D.J. Gittings, N.M. Elkassabany, J. Liu, C.L. Nelson, and A.F. Kamath, Preoperative Risk Factor Score Predicts Malnutrition in Total Joint Arthroplasty Patients. J Surg Orthop Adv, 2019. 28(2): p. 97-103.

19. Fryhofer, G.W., M. Sloan, and N.P. Sheth, Hypoalbuminemia remains an independent predictor of complications following total joint arthroplasty. J Orthop, 2019. 16(6): p. 552-558.

20. Ondeck, N.T., M.C. Fu, R.P. McLynn, P. Bovonratwet, R. Malpani, and J.N. Grauer, Preoperative laboratory testing for total hip arthroplasty: Unnecessary tests or a helpful prognosticator. J Orthop Sci, 2019.

21. Galea, V.P., P. Rojanasopondist, J.W. Connelly, C.R. Bragdon, J.I. Huddleston, 3rd, L.H. Ingelsrud, H. Malchau, and A. Troelsen, Changes in Patient Satisfaction Following Total Joint Arthroplasty. J 
Arthroplasty, 2019.

22. Sodhi, N., H.K. Anis, L.J. Garbarino, P.A. Gold, S.M. Kurtz, C.A. Higuera, M.S. Hepinstall, and M.A. Mont, Have We Actually Reduced Our 30-Day Short-Term Surgical Site Infection Rates in Primary Total Hip Arthroplasty in the United States? J Arthroplasty, 2019. 34(9): p. 2102-2106.

23. Shetty, T., J.T. Nguyen, A. Wu, M. Sasaki, E. Bogner, A. Burge, T. Cogsil, E.U. Kim, K. Cummings, E.P. Su, and S. Lyman, Risk Factors for Nerve Injury After Total Hip Arthroplasty: A Case-Control Study. J Arthroplasty, 2019. 34(1): p. 151-156.

24. Bjerregaard, L.S., M. Homilius, P. Bagi, T.B. Hansen, and H. Kehlet, Indwelling urinary catheterisation may increase risk of complications in hip and knee arthroplasty. Dan Med J, 2019. 66(4).

25. Lawrie, C.M., A.C. Ong, V.H. Hernandez, S. Rosas, Z.D. Post, and F.R. Orozco, Incidence and Risk Factors for Postoperative Urinary Retention in Total Hip Arthroplasty Performed Under Spinal Anesthesia. J Arthroplasty, 2017. 32(12): p. 3748-3751.

26. Halawi, M.J., N. Caminiti, M.P. Cote, A.D. Lindsay, and V.J. Williams, The Most Significant Risk Factors for Urinary Retention in Fast-track Total Joint Arthroplasty are latrogenic. J Arthroplasty, 2019. 34(1): p. 136-139.

27. Zeng, W.N., K. Zhou, Z.K. Zhou, B. Shen, J. Yang, P.D. Kang, and F.X. Pei, Comparison between drainage and non-drainage after total hip arthroplasty in Chinese subjects. Orthop Surg, 2014. 6(1): p. 28-32.

28. Hou, N., F. Jing, W. Rong, D.W. He, J.J. Zhu, L. Fang, and C.J. Sun, [Meta analysis of the efficacy and safety of drainage after total hip arthroplasty]. Zhonghua Yi Xue Za Zhi, 2017. 97(21): p. 1668-1672.

29. Xu, H., J. Xie, Y. Lei, Q. Huang, Z. Huang, and F. Pei, Closed suction drainage following routine primary total joint arthroplasty is associated with a higher transfusion rate and longer postoperative length of stay: a retrospective cohort study. J Orthop Surg Res, 2019. 14(1): p. 163.

30. Di Benedetto, P., A. Zangari, S. Magnanelli, V. Cainero, A. Beltrame, R. Gisonni, and A. Causero, Heterotopic Ossification in Primary Total Hip Arthroplasty: which is the role of drainage? Acta Biomed, 2019. 90(1-s): p. 92-97.

31. Basques, B.A., E.C. Gardner, J.O. Toy, N.S. Golinvaux, D.D. Bohl, and J.N. Grauer, Length of Stay and Readmission After Total Shoulder Arthroplasty: An Analysis of 1505 Cases. Am J Orthop (Belle Mead NJ), 2015. 44(8): p. E268-71.

32. DeMik, D.E., N.A. Bedard, S.B. Dowdle, J.M. Elkins, T.S. Brown, Y. Gao, and J.J. Callaghan, Complications and Obesity in Arthroplasty-A Hip is Not a Knee. J Arthroplasty, 2018. 33(10): p. 32813287.

33. Hannon, C.P., T.C. Keating, J.K. Lange, B.F. Ricciardi, B.S. Waddell, and C.J. Della Valle, Anesthesia and Analgesia Practices in Total Joint Arthroplasty: A Survey of the American Association of Hip and Knee Surgeons Membership. J Arthroplasty, 2019.

34. Kuchalik, J., A. Magnuson, A. Lundin, and A. Gupta, Local infiltration analgesia or femoral nerve block for postoperative pain management in patients undergoing total hip arthroplasty. A randomized, double-blind study. Scand J Pain, 2017. 16: p. 223-230. 
Figures

Initial dataset 611 patients 616 hips between January 2014 to January 2019

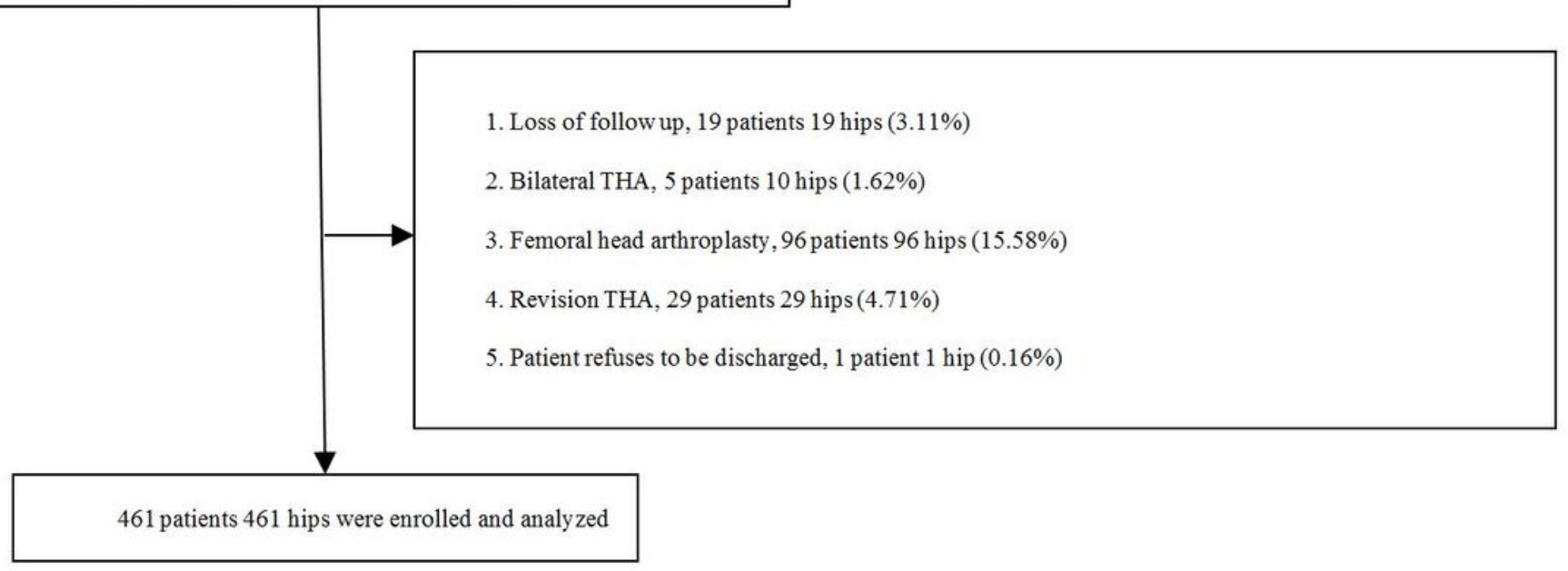

\section{Figure 1}

The flow diagram illustrates patient selection undergoing primary THA. 

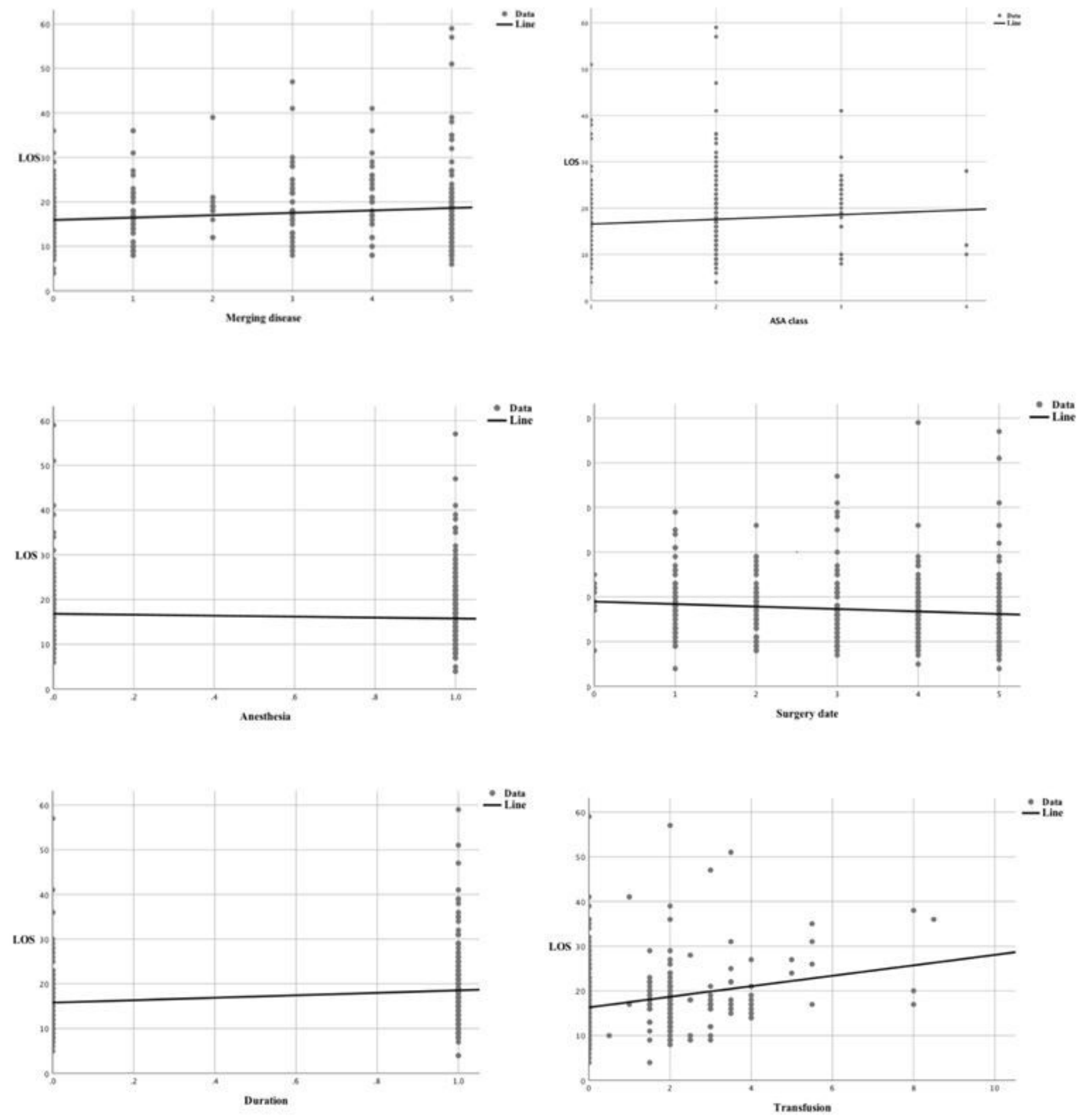

Figure 2

Regression curves.

\section{Supplementary Files}

This is a list of supplementary files associated with this preprint. Click to download. 
- Coverletter.docx

- JOSRManuscript.pdf

- Titlepage.docx 